\title{
Planck-scale quintessence and the physics of structure formation
}

\author{
Constantinos Skordis and Andreas Albrecht \\ Department of Physics, UC Davis, One Shields Ave, Davis CA 95616
}

\begin{abstract}
In a recent paper we considered the possibility of a scalar field providing an explanation for the cosmic acceleration. Our model had the interesting properties of attractor-like behavior and having its parameters of $O(1)$ in Planck units. Here we discuss the effect of the field on large scale structure and CMB anisotropies. We show how some versions of our model inspired by "brane" physics have novel features due to the fact that the scalar field has a significant role over a wider range of redshifts than for typical "dark energy" models. One of these features is the additional suppression of the formation of large scale structure, as compared with cosmological constant models. In light of the new pressures being placed on cosmological parameters (in particular $H_{0}$ ) by CMB data, this added suppression allows our "brane" models to give excellent fits to both CMB and large scale structure data.
\end{abstract}

PACS Numbers : 98.80.Cq, 95.35+d

\section{INTRODUCTION}

Current evidence that the expansion of the Universe is accelerating [1], if confirmed, requires dramatic changes in the field of theoretical cosmology. Until recently, there was strong prejudice against the idea that the Universe could be accelerating. There simply is no compelling theoretical framework that could accommodate an accelerating universe. Since the case for an accelerating universe continues to build (see for example [2]), attempts have been made to improve the theoretical situation, with some modest success. Still, major "fine tuning" problems remain.

All attempts to account for acceleration [3-31] introduce a new type of matter (the "dark energy" or "quintessence") with an equation of state $p_{\phi}=w_{\phi} \rho_{\phi}$ relating pressure and energy density. Values of $w_{\phi} \leq-0.6$ today are preferred by the data [32] and in many models $w_{\phi}$ can vary over time. (In this framework, a plain cosmological constant is the case where $w_{\phi}=-1$ independent of time.) Most models with a varying $w_{\phi}$ are based on a simple scalar field with a specific potential in the Lagrangian. In addition to the standard ingredients fed into the Friedman-Robertson-Walker (FRW) cosmology, one introduces a scalar field which in general has a varying $w_{\phi}$. If the value of $1+3 W_{\phi} \Omega_{\phi}$ becomes negative (with the assumption that the photon and neutrino contributions to the total density of the universe is small compared to the rest of the mass-energy) the universe enters an era of accelerated expansion.

The evolution of spatially homogeneous scalar fields in an FRW cosmology has a long history in the context of cosmic inflation, but the inflaton fields do not play a significant role today. In the last two years scalar fields have been considered which can produce an accelerated expansion in the present epoch This area has been stimulated by the growing evidence for cosmic acceleration today. The additional scalar field matter is known as "quintessence" or "dark energy". In the early models one had to fine-tune the initial conditions of the field in order to get an accelerated expanding universe today, a feature which is very undesirable. Later on the very interesting category of "tracker" quintessence models were created, in which the field gives the desired behavior independently of initial conditions. Still one had to introduce a small scale into the Lagrangian in order to achieve this. One way forward with these models is to try and construct a specific explanation for these small parameters 22,29.

In a recent paper [19] we discussed a class of quintessence models which like the "tracker" models, would produce the desired effect of an accelerated universe, independently of the initial conditions. The model was based on the pure exponential potential which was known to possess attractor solutions 33 39. However, the pure exponential however, in the attractor regime, cannot produce a realistic accelerated expanding universe. In our previous paper we showed that when the exponential potential is modified by a polynomial prefactor, we can keep the attractor solutions during most of the history of the universe (and therefore the independence from the initial conditions) while at the same time producing an accelerating scale factor today. The new nice feature of the model, not found previously in other models, was that all the parameters involved were $O(1)$ in Planck units. Since then, a number of authors taken this idea in interesting directions [40,41], which adds to the case for pursuing the observable effects of our model on cosmic structure. (We also note that yet another class models using simple Planck-scale physics have been proposed (42).

In this paper we consider the evolution of small perturbations for the former potential and discuss the implications on the Cosmic Microwave Background (CMB) and structure formation. In particular the recent Boomerang and Maxima data suggest that the Hubble constant might be larger than the so far accepted value of $65 \mathrm{kms}^{-1} \mathrm{Mpc}^{-1}$. 
In this case if one normalizes the data to COBE, the standard $\Lambda$-CDM model doesn't do that well for the observed matter power spectrum. The former potential seems to do slightly better. One of our important results comes from a modification to the potential reported in [19], inspired by "brane" physics which results in a number of novel features. One of these features is a much better joint fit to the matter power spectrum and CMB anisotropy power.

The structure of this paper is as follows. First we review the evolution of scalar fields in an FRW cosmology. We discuss in particular the two mentioned potentials (the one from 19] and the "brane" variation) emphasizing the relevant physics and noting the differences between the two of them and between other models. Then we present the evolution of small perturbations for the two fields at hand and discuss the various effects. The evolution of perturbations is different in the two models which leads to considerable differences in the power spectra. The effect of the additional dark energy in the universe on the matter power spectrum is discussed next. We consider parameters of the models that give good $\sigma_{8}$ 's and fit the newly published decorrelated data of Hamilton et.al. [52,53]. Following that we focus on the related physics that affect the CMB and also discuss the difference between the two models and $\Lambda$ models. A final section gives our conclusions.

\section{EVOLUTION OF THE BACKGROUND}

Let us review the background evolution for a scalar field cosmology. This section does not introduce anything new (except the final part which introduces the new potential) and is merely intended for the new readers not familiar with the subject. We use a $(+---)$ metric signature, with Greek indices running from 0 to 3 and Latin indices (spatial) from 1 to 3 . Index free 3 -vectors are denoted as $\vec{x}$ and 4 -vectors as $\underline{x}$. Unless otherwise told, all units will be Planckian with $M_{p}=\frac{1}{\sqrt{k_{e}}} \equiv(8 \pi G)^{(-1 / 2)}=1, k_{e}$ being Einstein' constant and $M_{p}$ being the Planck mass. Moreover we assume that the zeroth order cosmology is flat FRW in the conformal synchronous gauge.

\section{A. Cosmological scalar field evolution}

The point of departure is the action functional for the scalar field and gravity,

$$
S\left[g_{\alpha \beta}, \phi\right]=\int d^{4} x \sqrt{-g}\left\{\frac{R}{2}-\frac{1}{2} \phi_{, \mu} \phi^{, \mu}+V(\phi)\right\}
$$

Since the background metric has been fixed by our assumptions we can use $\sqrt{-g}=a^{4}$ and $R=-6 \frac{\ddot{a}}{a^{3}}$. Moreover the assumption of homogeneity and anisotropy forces the spatial derivatives of the field to zero, $\phi_{, i}=0$. The action simplifies, and takes the form of one particle Lagrangian mechanics in two dimensions with coordinates $a(\tau)$ and $\phi(\tau)$.

$$
S[a(\tau), \phi(\tau)]=\int d \tau\left\{3 \dot{a}^{2}-\frac{1}{2} a^{2} \dot{\phi}^{2}+a^{4} V(\phi)\right\}
$$

The canonical momenta are $p_{a}=6 \dot{a}$ and $p_{\phi}=a^{2} \dot{\phi}$. The constraint $H=0$ gives the first Friedman equation

$$
3\left(\frac{\dot{a}}{a^{2}}\right)^{2}=\frac{1}{2 a^{2}} \dot{\phi}^{2}+V(\phi)
$$

and therefore the field density is

$$
\rho=\frac{1}{2 a^{2}} \dot{\phi}^{2}+V(\phi)
$$

Variation w.r.t. the field $\phi(\tau)$ gives the field equation of motion

$$
\ddot{\phi}+2 \frac{\dot{a}}{a} \dot{\phi}+a^{2} V_{, \phi}=0
$$

where ()$_{,}$denotes derivative with respect to $\phi$.

Finally variation w.r.t. the scale factor $a(\tau)$ gives the second Friedman equation

$$
6 \frac{\ddot{a}}{a^{3}}=-\frac{1}{a^{2}} \dot{\phi}^{2}+4 V=\rho-3 P
$$


from which we get the field pressure as

$$
P=\frac{1}{2 a^{2}} \dot{\phi}^{2}-V(\phi)
$$

If we want our scalar field to accelerate the universe we need the field to provide the dominant form of energy density and at the same time have negative pressure. In order to accelerate the universe a necessary and sufficient condition is that the deceleration parameter $q$ given by

$$
q=-\frac{a \ddot{a}}{\dot{a}^{2}}=\frac{1}{2}\left(1+3 w_{\phi} \Omega_{\phi}+\Omega_{r}\right)
$$

be negative or equivalently $P_{\phi}<-H^{2}$ (In the last equation the dot denotes a derivative with respect to real time).

\section{B. The pure exponential potential}

One very interesting potential is the exponential $V=V_{0} e^{-\lambda \phi}$. This potential has been shown to have attractor solutions [33] 34], that is regardless of initial conditions, the field eventually scales like the dominant matter component. For a detailed discussion see [39].

If the dominant component scales as $\rho_{n}=\rho_{0}\left(\frac{a_{0}}{a}\right)^{n}$ then the scalar field approaches an attractor solution and its fractional density is given by $\Omega_{\phi}=\frac{n}{\lambda^{2}}$. This is a special case of scaling a field - a classification of such fields can be found in [?].
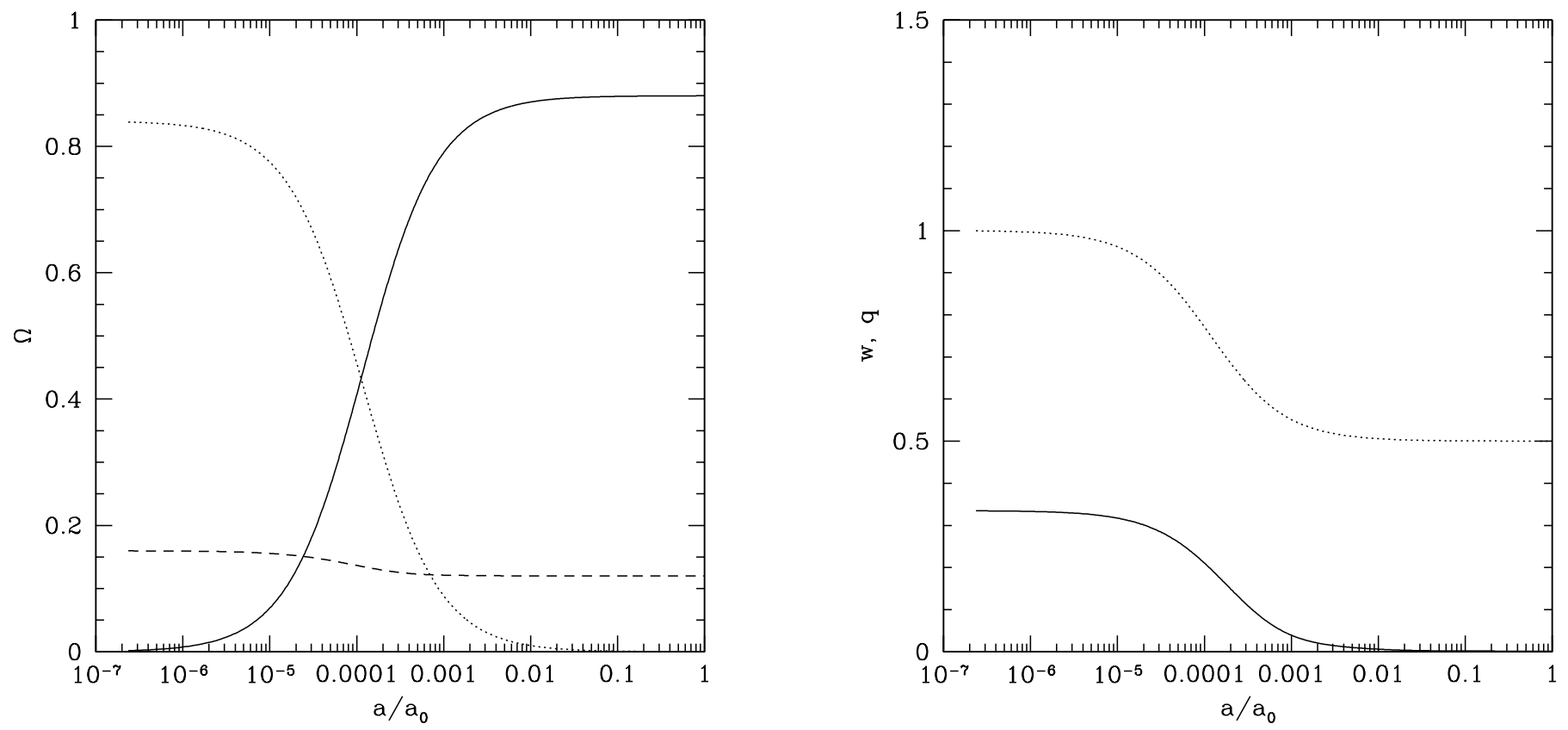

FIG. 1. LEFT: Fractional densities $\Omega$ as a function $a$ for the exponential potential. The solid curve corresponds to matter with $\Omega_{m} \approx 0.88$ today, the dotted curve corresponds to radiation $T=2.726 \mathrm{~K}$ today and the dashed curve corresponds to the scalar field with $\Omega_{\phi} \approx 0.12(\lambda=5)$ today. Radiation-to-matter transition is around $a=10^{-4}$ and $a=1$ today.

RIGHT: Evolution of the equation of state parameter $w_{\phi}$ and the deceleration parameter $q_{\phi}$ as a function of a for the same model. The solid curve corresponds to $w$ and the dotted one to $q$. The attractor causes $w_{\phi}$ to simply mimics the $w$ of the dominant form of matter so $q_{\phi}$ can never be negative in the attractor regime of a pure exponential potential.

During the attractor era the relevant physical quantities are

$$
\begin{array}{r}
\rho_{\phi}=\frac{6}{6-n} V=\frac{3}{n a^{2}} \dot{\phi}^{2} \\
\frac{\dot{a}}{a}=\left(\frac{2}{n-2}\right) \frac{1}{\tau}
\end{array}
$$


where $n=3(1+w)$.

Figure 1 shows the evolution of such a field with the scale factor. Notice the change of the fractional density in the field from the radiation to the matter era. Since $w$ simply mimics the $w$ of the dominant matter, it is impossible to get an accelerating universe in the attractor era. It is possible to avoid the attractor if one chooses $\lambda<\sqrt{n}$. In that case the attractor is not there but the field will have its own scaling behavior with $w_{\phi}=\frac{\lambda^{2}}{3}-1$. It is also possible to choose initial conditions sufficiently far from the attractor solution that the attractor has not been reached even today 43 .

\section{Modifying the exponential potential}

The attractor behavior of the exponential is appealing, and it would be nice if we could modify it in such a way as to keep this behavior for most of the history of the universe and at the same time get an accelerated universe today. Indeed we showed in a previous paper 19 that this is possible by including a prefactor in front of the exponential $V=V_{p}(\phi) e^{-\lambda \phi}$. The effect of the prefactor is to introduce a local minimum in the exponential such that the field gets trapped into it. After the field gets trapped it starts behaving like a cosmological constant and the universe eventually enters an era of accelerated expansion. In our previous paper we used a polynomial prefactor. The full potential then takes the form

$$
V=V_{0}\left[(\phi-B)^{2}+A\right] e^{-\lambda \phi}
$$

Through this paper we refer to this potential as the "AS" model.

The left panel of Fig. 2 shows the cosmological evolution of densities in an AS model. One noticeable feature of this model is the breakaway from the attractor behavior at late times. This will be important for structure formation as the field density becomes very small when structure begins to form and so it doesn't affect structure formation much. Lastly we should note that trapping takes place independently of initial conditions of the quintessence field in the very early Universe. This is because the Universe is drawn into the attractor at early times. Since the field is in the attractor solution by the time it approaches the local minimum there is no memory of the initial conditions. There is a variety of possible behaviors at the local minimum. If the minimum is very shallow however the field can roll through rather than be trapped. It is also possible to choose parameters which remove the local minimum, but the field still lingers long enough to cause an era of accelerated expansion. For trapping a necessary condition is $A \lambda^{2}<1$. The various behaviors and their corresponding parameter ranges have been investigated in [44].

Instead of choosing a polynomial prefactor it is possible to have the desired accelerated expansion by using a different function $V(\phi)$. An oscillating term for example could do the job [31]. Here we give another form which can accelerate the universe円.

$$
V=\left[\frac{C}{(\phi-B)^{2}+A}+D\right] e^{-\lambda \phi}
$$

Such a potential could arise from the various brane models as a Yukawa-like interaction between branes [46] (we will call it throughout this paper the "Brane" model).

The right panel of Fig. 2 2 shows the evolution of densities with the Brane potential. During radiation era it behaves like the AS potential. During the matter era however it retains significant density unlike the AS potential. This has a significant impact on both structure formation (which is suppressed) and the CMB as we shall see later.

To understand why there is such a difference we plot the equation of state parameter $w$ with the scale factor in Fig. 3. In the case of the AS model, soon after the field enters the matter era attractor where $w$ should go eventually to 0 , the field instead rushes toward the minimum of the potential and then undergoes damped oscillation. During that time the energy density of the field falls of significantly faster than the than energy density of the pressureless matter. This behavior corresponds with a brief rise in $w(a)$. Due to damping from the expansion however, the oscillations eventually stop, the field settles in the minimum and starts behaving like a cosmological constant. This is shown by the subsequent oscillations of $w$ with decreasing amplitude until $w$ goes to -1 .

In the case of the Brane model the potential has a much sharper minimum. In that case the field stays longer in the attractor regime until suddenly it gets trapped in the minimum without oscillating. The effect is that $w$ remains zero during most of the period of structure formation (with the field in the matter attractor regime) and then goes to

\footnotetext{
${ }^{1}$ This model was developed jointly with J. Weller 45
} 

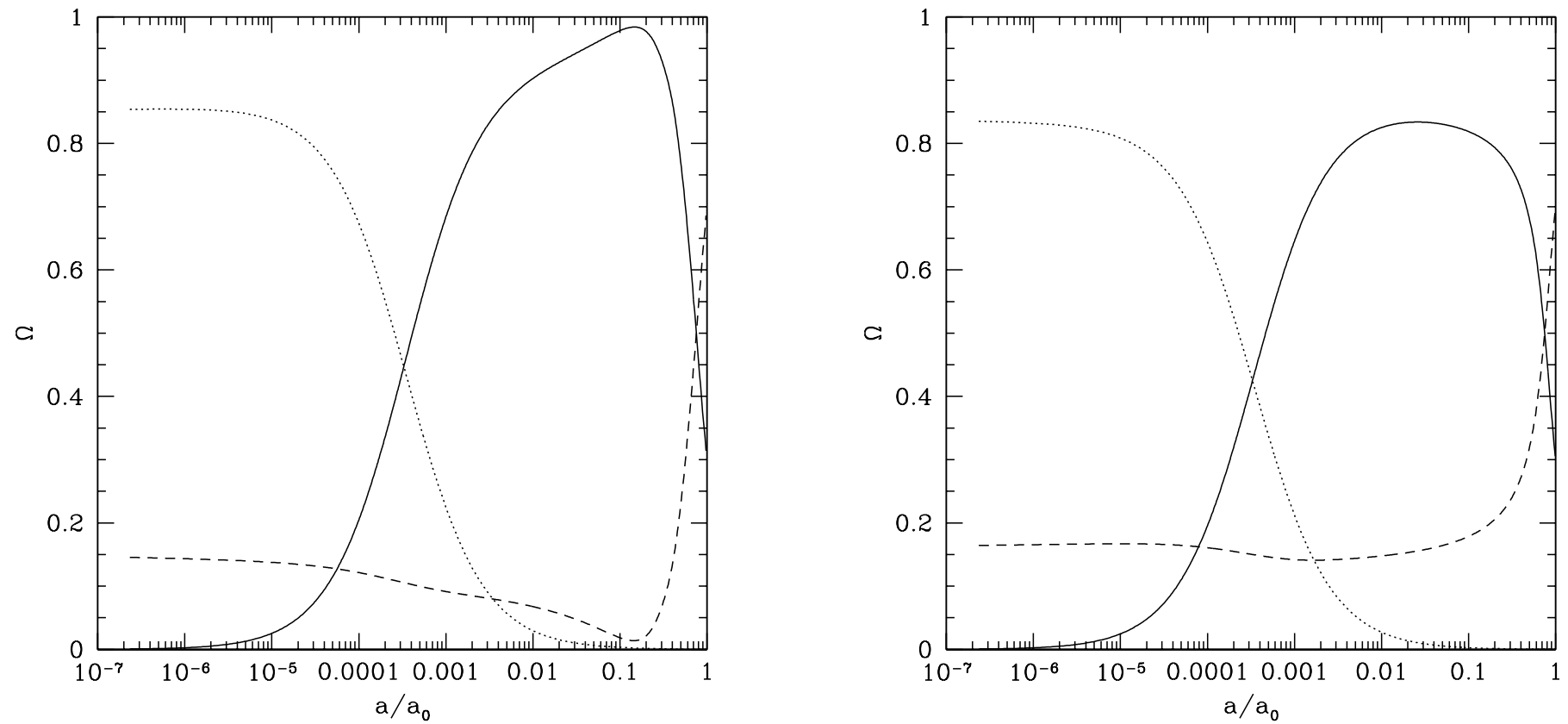

FIG. 2. Solutions obtained by including a $V_{p}$ factor in the potential. We show $\Omega_{\phi}(a)$ (dashed), $\Omega_{\text {matter }}(a)$ (solid) and $\Omega_{\text {radiation }}(a)$ (dotted). Radiation-to-matter transition is around $a=10^{-4}$ and $a=1$ today. Both models have $h=0.65$, $\Omega_{\phi}=0.7$ and $\Omega_{m}=0.247$ today.

LEFT: The AS model with $\lambda=5, B=54.4057, A=.01$. Notice the behavior of the field at late times where $\Omega_{\phi}$ goes very near to zero. That is the period of structure formation so the field doesn't affect structure formation much.

RIGHT: The Brane model with $\lambda=5, B=56.10425, A=.01, C=1$ and $D=0.1$. Notice that the behavior of the field at late times is different from the AS model in the sense that the field retains a significant amount of energy density. This behavior affects structure formation as well as the CMB.

-1 directly to start accelerating the universe. This allows the field to retain the significant amount of energy density seen previously. Moreover because of this, the Brane model starts accelerating the universe later than the AS model.

Another question concerning both potentials above is the stability of the local minimum under quantum tunneling. It has been shown however that tunneling is negligible which renders the minimum in both potentials effectively stable [15].

Finally we should say that the tightest constraint comes from requiring that $\Omega_{\phi}$ not be too large during nucleosynthesis 47 (at $a \approx 10^{-10}$ ). For $\Omega_{\phi}(1 \mathrm{MeV}) \leq 0.1$ we get that $\lambda \geq 6.3$. The models shown in Fig. 2 do not obey this constraint because they were chosen to dramatize the different behaviors. When comparing with data, we use more realistic parameters.

\section{DENSITY PERTURBATIONS}

To make connection with the real universe one has to consider the growth of small perturbations about an FRW metric. For extensive reviews see for example 48 51]. Here we adopt the conventions of Ma and Bertschinger [51]. We start with a line element given by

$$
d s^{2}=a^{2}(\tau)\left[-d \tau^{2}+\left(\delta_{i j}+h_{i j}\right) d x^{i} d x^{j}\right]
$$

The metric perturbation is split into scalar, vector and tensor modes as usual.

\section{A. Perturbations in the scalar field}

Here we give the relevant equations for the perturbations in the quintessence. For an extensive review of scalar field perturbations in cosmology see for example [49,50] and for the first applications in the case of quintessence [5, 8]. 

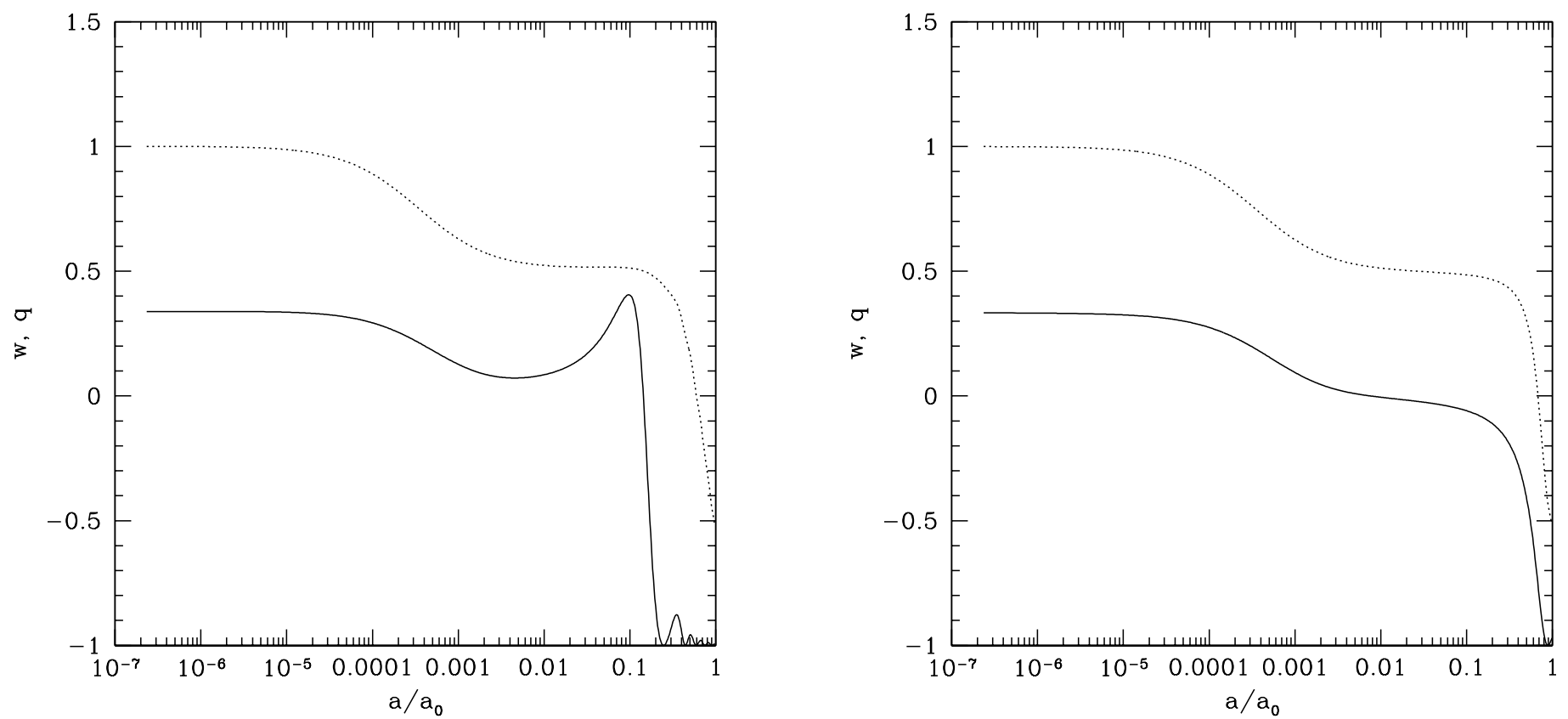

FIG. 3. The equation of state parameter $w$ (solid) and deceleration parameter $q$ (dotted) for the same two models of Fig. 2. On the left we have the AS model and on the right the Brane model. The difference of the two models seen in Fig. 8 is seen again here in $w$.(see the text for more details) Acceleration begins around $a=0.7-0.9$ when $q$ becomes negative.

To include scalar fields into the perturbations lets write the field as $\phi(\underline{x})=\phi_{0}(\tau)+\phi_{1}(\underline{x})$ with $\phi_{1}<<\phi_{0}$, where $\phi_{0}(\tau)$ represents the zeroth order homogeneous field i.e. $\nabla \phi_{0}=0$. We can then form the stress-energy tensor from which we can read the perturbed density $\rho_{1}$, pressure $P_{1}$ and velocity divergence $\theta$ of the field. It is important to note that the shear is zero independently of the form of the potential. The quantities of interest in $k-$ space are

$$
\begin{aligned}
\rho_{1} & =\frac{1}{a^{2}} \dot{\phi}_{0} \dot{\phi}_{1}+V_{, \phi} \phi_{1} \\
P_{1} & =\frac{1}{a^{2}} \dot{\phi}_{0} \dot{\phi}_{1}-V_{, \phi} \phi_{1} \\
\theta & =\frac{k^{2} \phi_{1}}{\dot{\phi}_{0}}
\end{aligned}
$$

The field perturbation also obeys a Klein-Gordon equation

$$
\ddot{\phi_{1}}+2 \frac{\dot{a}}{a} \dot{\phi}_{1}+\left(k^{2}+a^{2} V_{, \phi \phi}\right) \phi_{1}+\frac{1}{2} \dot{h} \dot{\phi}_{0}=0
$$

The above 2nd order equation is equivalent to the two 1st order equations (29) of 151. One has to keep in mind however that in general (as noted also in [30]), the density perturbations in the field are not adiabatic i.e. $\frac{P_{1}}{\rho_{1}}$ is not in general equal to $\frac{d P}{d \rho}$. We shall use the symbols $c_{w}$ and $c_{s}$ for $c_{w}^{2}=\frac{P_{1}}{\rho_{1}}$ and $c_{s}^{2}=\frac{d P}{d \rho}$, the later being the adiabatic speed of sound. If the field is adiabatic then the two quantities become equal $c_{w}=c_{s}$. The above point is important for understanding why even if quintessence has the same equation of state as CDM it nevertheless does not clump. It all boils down to how the Jeans length is defined. In the case of CDM, the Jeans length is zero by definition, contrary to quintessence where it can be as large as the horizon. This is because the Jeans length must be defined in terms of $c_{w}$ and not $c_{s}$ to include the effect of entropy perturbations. In the case of quintessence it is exactly this effect which prevents clumping. Basically, the quintessence field is able to have a non-zero pressure perturbations even when $c_{s}=0$, and the pressure will resist gravitational collapse.

Another thing to note about the field equation (17) is the physical meaning of the various terms. The Hubble expansion provides a time-dependent "drag" term and the potential a time dependent "mass" term. The last term gives the gravitational sources for the field perturbations. 


\section{B. Growing super-horizon perturbations in the radiation era}

In our model, deep in the radiation era we can assume that the field is in the attractor and that the potential is a pure exponential. In this case we can use the analysis of Ferreira and Joyce 39] to get the initial conditions. We should note that the initial conditions given here are curvature initial conditions.

We can use (10) with $n=4$ for the radiation era. Then we have for Quintessence

$$
\begin{aligned}
\rho_{\phi} & =3 V=\frac{3}{4} \dot{\phi}_{0}{ }^{2} \\
\frac{\dot{a}}{a} & =\frac{1}{\tau} \\
\Omega_{\phi} & =\frac{4}{\lambda^{2}} \\
\delta_{\phi} & =\frac{\lambda}{3}\left(\tau \dot{\phi_{1}}-\phi_{1}\right) \\
c_{w \phi}^{2} & =\frac{\tau \dot{\phi_{1}}+\phi_{1}}{\tau \dot{\phi}_{1}-\phi_{1}} \\
\tau^{2} \ddot{\phi}_{1}+2 \tau \dot{\phi_{1}} & +\left[(k \tau)^{2}+4\right] \phi_{1}+\frac{2}{\lambda} \tau \dot{h}=0
\end{aligned}
$$

Assuming that only photons and quintessence are dominant during that period we find for the gravitational perturbation $h$

$$
\tau^{2} \ddot{h}+\tau \dot{h}+6 \Omega_{\gamma} \delta_{\gamma}+3\left(1+3 c_{w \phi}^{2}\right) \Omega_{\phi} \delta_{\phi}=0
$$

For superhorison perturbations we assume $k \tau<<1$ and therefore to first order $\theta_{\gamma}=0$. Therefore

$$
\delta_{\gamma}=-\frac{2}{3} h
$$

Combining the last six equations (for $k^{2}=0$ ) we get

$$
\begin{aligned}
\tau^{2} \ddot{h}+\tau \dot{h}-4\left(1-\frac{4}{\lambda^{2}}\right) h+\frac{8}{\lambda}\left(2 \tau \dot{\phi}_{1}+\phi_{1}\right) & =0 \\
\tau^{2} \ddot{\phi}_{1}+2 \tau \dot{\phi}_{1}+4 \phi_{1}+\frac{2}{\lambda} \tau \dot{h} & =0
\end{aligned}
$$

Let $h=C \tau^{m}$ and $\phi_{1}=D \tau^{m}$. Then

$$
m= \pm 2, \quad m=\frac{-1 \pm \sqrt{\frac{64}{\lambda^{2}}-15}}{2}
$$

The growing physical mode is the one proportional to $\tau^{2}$. For the coefficients $C$ and $D$ we get (a result also found by Ferreira and Joyce)

$$
D=-\frac{2}{5 \lambda} C
$$

This gives

$$
\delta_{\phi}=\frac{4}{15} \delta_{c}
$$

Note that the above result gives $c_{w}^{2}=3 \neq c_{s}^{2}=w=\frac{1}{3}$ so quintessence is not adiabatic even if $\dot{w}=0$. 


\section{Initial Conditions}

From the above analysis we can form the initial conditions following the procedure in Ma and Bertschinger. The initial conditions for all the standard quantities remain the same. The two additional initial conditions for quintessence are

$$
\phi_{1}=-\frac{2}{5 \lambda} h, \quad \dot{\phi}_{1}=-\frac{2}{5 \lambda} \dot{h}
$$

Even if we don't use the correct initial conditions for the field, as a property of the growing curvature mode, the correct evolution of the field density contrast will be reached quite rapidly and the initial conditions given above will be valid.

\section{STRUCTURE FORMATION}

First we investigate the growth of structure with time using the density perturbations as a guide. Then based on that investigation we give the resulting matter power spectrum and explain its form.

\section{A. The growth of structure}

One intuitive and important quantity is the dimensionless growth rate for the cold dark matter,

$$
n_{\mathrm{eff}}=\tau \frac{\dot{\delta_{c}}}{\delta_{c}} .
$$

This quantity give an instantaneous measure of the growth rate of structure. The time evolution of $n_{\text {eff }}$ is shown for a number of models on the left panel of Fig. 1. Initially, outside the horizon, $n_{\mathrm{eff}}=2$ completely independent of the model of structure formation(for a flat universe). This is due to the fact that because of causality the form of the background energy density is not relevant, only the amount. After entering the horizon, the growth of structure is suppressed during the radiation era simply because dark matter is extremely sub-dominant. During the matter era, dark matter becomes dominant again and in the case of an SCDM model, $n_{\text {eff }}$ eventually reaches the value of 2 again. For a $\Lambda \mathrm{CDM}$ model however, $n_{\text {eff }}$ after growing for a while, it eventually drops down to zero. This happens when the universe enters the accelerating era where the perturbations leave the horizon and structure stops forming.

The two quintessence models considered here have a similar effect to a $\Lambda$ CDM model. The only difference is the existence of a significant amount of dark energy during the matter era. This results in a suppression of structure formation since the amount of dark matter is less and therefore structure forms less efficiently. As stressed in section III.A, even when $w_{\phi}=0$ quintessence can have pressure perturbations which allow it to resist clumping.

This is not very important for the AS potential since if you notice from Fig. 2, $\Omega_{\phi}$ goes very close to zero before the accelerating era. Therefore in that model structure is just a little bit suppressed compared to a $\Lambda$ CDM model, but not by much.

For the Brane potential however the above effect is much stronger. In fact $\Omega_{\phi}$ stays quite significant during all of the matter era. Therefore for that potential, structure growth is even more suppressed as one can see from Fig. 4 . Not only do we have the acceleration era like before which suppresses structure anyway, we also have a significant amount of quintessence which doesn't cluster. This means that the amount of clustering matter is even less in this case so structure is even more suppressed.

\section{B. The matter power spectrum}

Based on the reasoning of the previous section, we can predict the form of the matter power spectrum. For the AS model we expect the power to be a bit less than for $\Lambda$ model with the same cosmological parameters. The Brane model should exhibit additional suppression. This is indeed the case as one can see in the right panel of Fig. 4 .

If we use a Hubble constant of $75 \mathrm{kms}^{-1} \mathrm{Mpc}^{-1}$, even though structure is suppressed for the Brane model, it fits the data very nicely. All of the models shown in the figure have $h=0.75, \Omega_{c}=0.297, \Omega_{b}=0.053, \Omega_{\phi}=0.65$ and some reionization with $\tau_{\mathrm{opt}}=0.1$. The corresponding CMB anisotropies are shown later in the right panel of Fig. 7 . The $\sigma_{8}$ 's 1.08,0.98 and 0.77 for $\Lambda \mathrm{CDM}$, AS and Brane potentials respectively. 

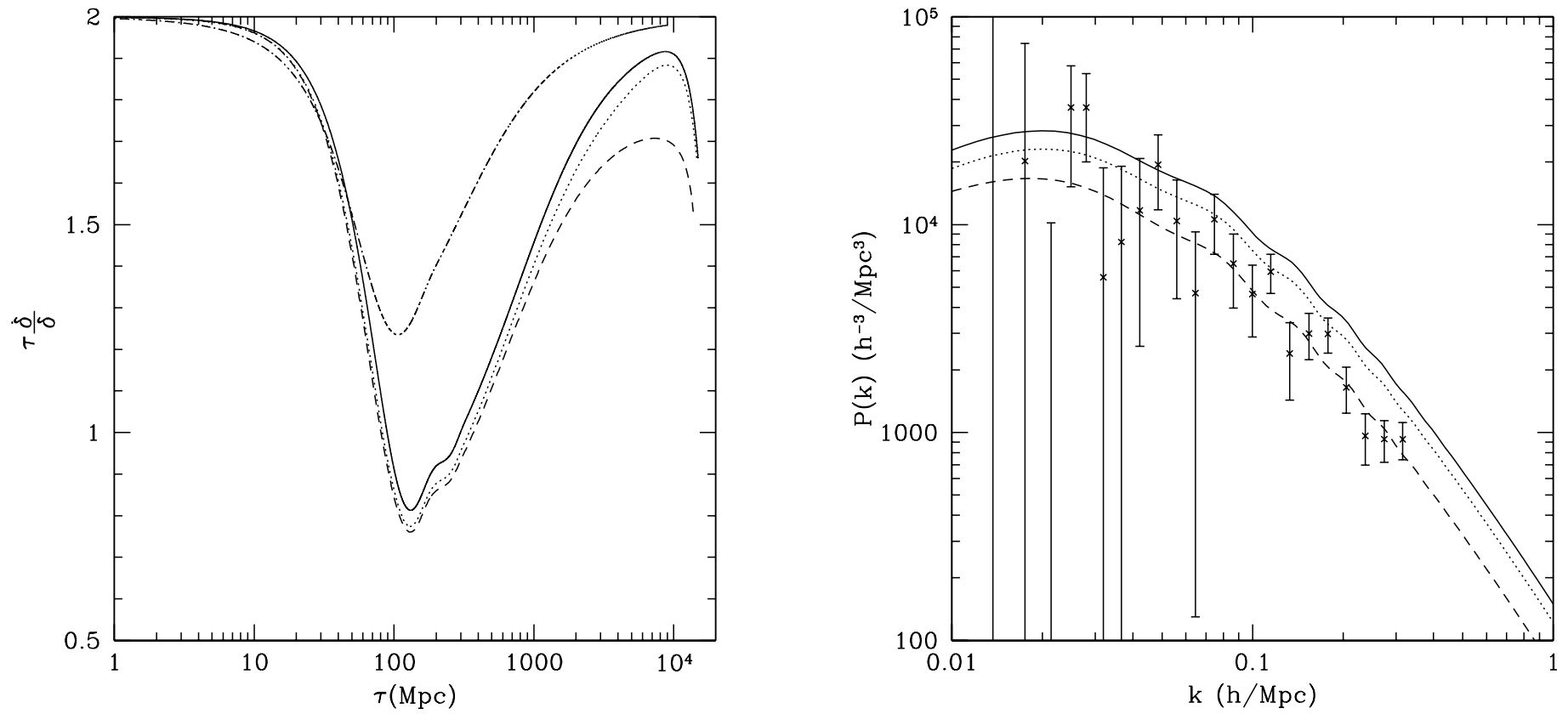

FIG. 4. LEFT: The effective dimensionless growth rate $n_{\text {eff }}$ for four different models at $k=0.1 M p c^{-1}$. At early times $n_{\text {eff }} \rightarrow 2$ for all models, due to the $\tau^{2}$ dependence of the growing superhorizon modes. Growth is suppressed in the radiation era $(\tau \approx 100 M p c)$ and in the $\phi$-era (today). In the matter era $\mathrm{sCDM}$ (dash-dotted) has the most growth, then $\Lambda$ CDM (solid), AS (dotted) and finally with the least growth the Brane (dashed). The last two are the same models shown in Fig. 2 .

RIGHT: The matter power spectrum normalized to COBE using the Bunn-White fitting formula. The plotted models are $\Lambda$ (solid), AS (dotted) Brane (dashed). All the models have $h=0.75, \Omega_{c}=0.297, \Omega_{b}=0.053$ and $\Omega_{\phi}=0.65$. Here we use more realistic quintessence parameters consistent nucleosynthesis. In particular for the AS model $\lambda=8, B=33.9627, A=0.01$ and for the Brane model $\lambda=8, B=35.13689, C=0.01$ and $D=0.01$. The data points are the decorrelated data of Hamilton et.al. [52,53]

It is not the goal of this paper to scan all of parameter space and compare a wide range of models, and we are not suggesting that any of the models in Fig. 4 are the best fitting model of their type. Figure 4 is intended to illustrate the physical differences of the different types of models by holding as many aspects as possible constant across the four models. Figure 1 also illustrates the somewhat surprising result that it is possible for models with significant contributions from the dark energy at early times (i.e.the Brane model) to provide a good fit to the data.

\section{THE COSMIC MICROWAVE BACKGROUND}

Lastly we give the CMB anisotropy power spectrum and investigate the various physical effects due to the field. We follow the analysis of $\mathrm{Hu}$ and Sugiyama 54 56. Following the common practice we divide the CMB anisotropies into primary and secondary. The primary anisotropies are the ones formed at the Last Scattering Surface (LSS), the secondary being the ones due to the subsequent cosmological evolution. Due to significant quintessence energy density at the LSS, the primary anisotropies are different compared with other models. In this section we drop sCDM from consideration and compare the remaining three models discussed above: the AS and Brane models, and the $\Lambda$ CDM model with similar parameters. Moreover, since we are only interested in the differences cause by quintessence, we keep other cosmological parameters $\left(\Omega_{b}, \Omega_{c}\right.$ and $\left.H_{0}\right)$ fixed.

\section{A. Primary anisotropies}

With the above parameters fixed, the only other parameter that can affect the primary anisotropies is the Hubble parameter during the time of LSS and earlier. Changing the Hubble parameter affects the heights of the peaks through three main effects, the driving effect, the doppler shift and Silk damping. 

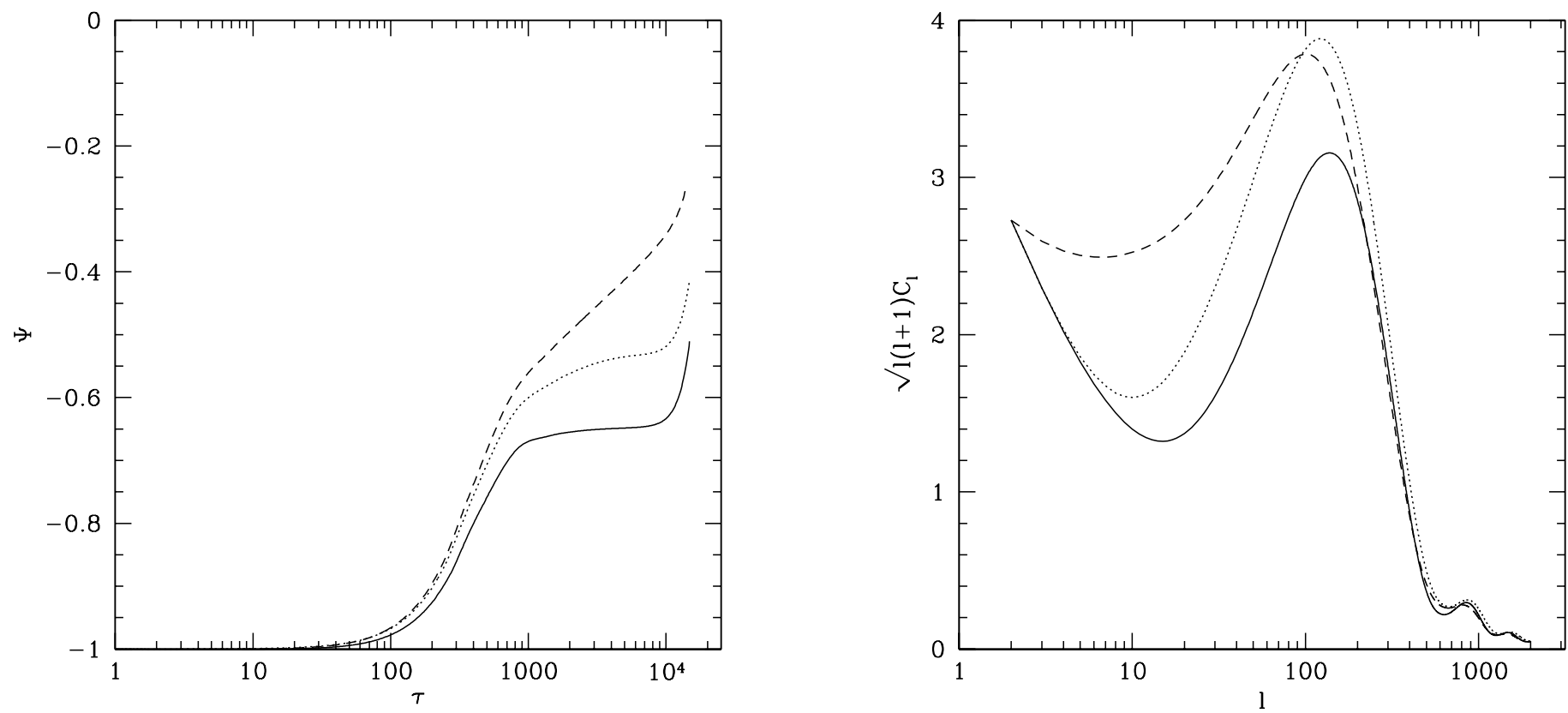

FIG. 5. LEFT: The Newtonian potential $\psi(\tau)$ at $k=0.01 M p c^{-1}$, normalized to -1 as $k \tau \rightarrow 0$. Shown are the same three models of Fig. 2: AS model (dotted), $\Lambda$ (solid), Brane model (dashed). Notice the very strong decay of the potential in the case of the Brane model, which leads to a very strong ISW effect.

RIGHT: An illustration of the ISW effect for the same models. All models have the same initial power spectrum. The strong ISW for the Brane model at COBE scales affects the normalization and reduces the final anisotropies.

Looking at fixed $a$ it is easy to show that the Hubble parameter in a quintessence model $H_{\phi}(a)$ is related to the Hubble parameter in a $\Lambda$ model $H_{\Lambda}(a)$ at the same $a$, by

$$
H_{\phi}=H_{\Lambda} \sqrt{\frac{1-\Omega_{\Lambda}}{1-\Omega_{\phi}}}
$$

Therefore, since for the quintessence models under consideration here $\Omega_{\phi}$ is quite significant during all the history of the universe(where as $\Omega_{\Lambda}$ isn't), the Hubble parameter in a quintessence universe will in general be larger than the one in a $\Lambda$ universe. The above statement is of course true only if we keep $\Omega_{0 m}, \Omega_{0 \phi}$ and $H_{0}$ fixed which is what we do for comparing the models.

The driving effect arises from a decaying Newtonian potential $\Psi$ before the LSS (see Fig. 5), as well as a dilation effect from a decaying space curvature $\Phi$ (not to be confused with $\phi$ which is the quintessence field). The Newtonian potential and the curvature can be written in terms of the other perturbations as

$$
\begin{aligned}
& \Phi=-\frac{3 a^{2} H^{2}}{2 k^{2}} \sum_{i}\left[\Omega_{i} \delta_{i}+\frac{3 a H}{k^{2}}\left(1+w_{i}\right) \Omega_{i} \theta_{i}\right] \\
& \Psi=\Phi-\frac{6 a^{2} H^{2}}{k^{2}}\left(\Omega_{\gamma} \sigma_{\gamma}+3 \Omega_{\nu} \sigma \nu\right)
\end{aligned}
$$

The effect of quintessence on the driving effect comes from the significant fractional densities of quintessence during the radiation and matter era. During the radiation era $\Omega_{\phi}$ is always greater than the on during matter era. This results in a greater difference of $H_{\phi}$ from $H_{\Lambda}$ in the radiation era than in the matter era. The result is both the Newtonian potential and curvature decay faster in the case of quintessence. The effect is an increased driving effect in the case of quintessence which increases the temperature anisotropies for all peaks. We should also note that the same effect occurs by having more species of relativistic particles around(e.g. more massless neutrinos), the reason being exactly the same. Moreover this is more significant for the Brane potential whose Hubble parameter is always greater that the one for the AS potential.

The velocity perturbation makes a contribution to the temperature anisotropies that is 90 degrees out of phase with the contribution from the density perturbation. The velocity is sub-dominant compared to the density which 

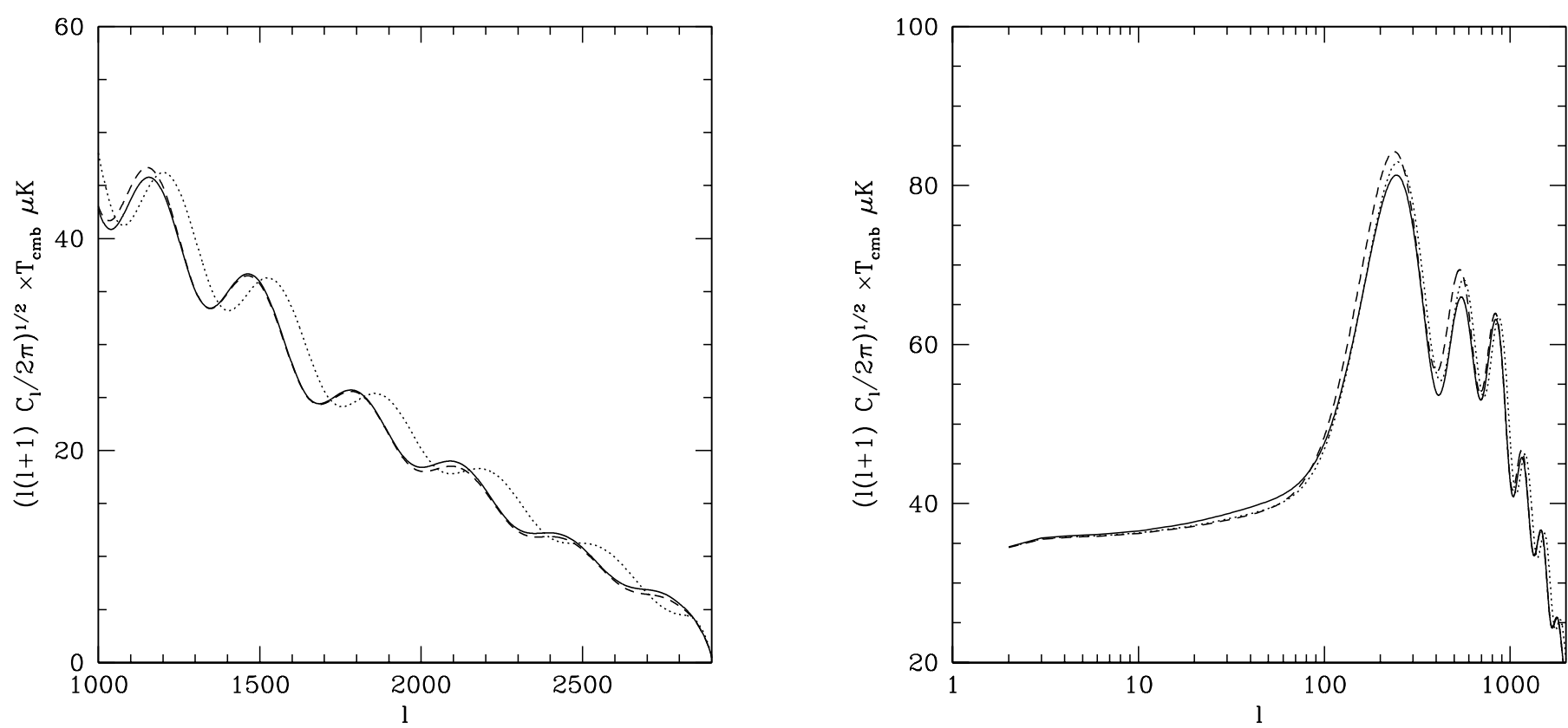

FIG. 6. LEFT: The effect of Silk damping on the total primary anisotropies for the same models as Fig.2 with the same initial power spectrum. In the case of quintessence, Silk damping is slightly more effective(see text). RIGHT: Primary temperature anisotropies for the same models, as would have been observed today if there was no other anisotropy source. The driving effect is observable on the first four peaks. The much higher 2nd peak for quintessence as well as the trough before it shows the Doppler effect.

makes the effect weak. There is however a small but observable difference between a $\Lambda$ model and quintessence. For quintessence even peaks as well as the troughs before them are slightly raised compared to a $\Lambda$ model. This causes the oscillations appear to be "fattened" up due to the modulation of the velocity with the density to get the final temperature anisotropy.

Finally we have Silk damping which is different in the three models. The damping coefficient $k_{D}$ is given by

$$
k_{D}^{-2}(a)=\frac{1}{6} \int_{0}^{a} \frac{1}{a^{4} H^{2} \tau^{\prime}} \frac{R^{2}+\frac{4}{5}(1+R)}{(1+R)^{2}} d a
$$

where $R=\frac{3 \rho_{b}}{4 \rho_{\gamma}}$ and $\tau_{\mathrm{opt}}$ is the optical depth. The damping coefficient is proportional to the Hubble parameter and is therefore smaller in the case of quintessence. This means that damping becomes more effective at smaller $k$ (larger scales) in the quintessence models. The effect will be to suppress the small scale temperature anisotropies more effectively. Since at large $k$ the driving effect eventually becomes unimportant, Silk damping eventually becomes stronger than the driving effect at small scales. This makes a $\Lambda$ model with equivalent cosmological parameters have larger anisotropies than quintessence even though quintessence has larger anisotropies in the first two peaks. The total primary anisotropies are shown in Fig.6

Let us now consider the secondary anisotropies which can change this picture quite a lot.

\section{B. Secondary anisotropies}

The most notable secondary anisotropy is the Integrated Sachs-Wolfe (ISW) effect. To dramatize this effect for pedagogical purposes we first consider the models from Fig. 2 which have a higher proportion of Quintessence than is allowed by nucleosynthesis (and an equally bad $\Lambda$ model).

The ISW comes from a decaying Newtonian potential after the LSS (see Fig. 5). The standard treatment breaks the ISW down to early and late ISW. In our case however we have an intermediate ISW as well.

The right panel of Fig. 5 shows ISW contribution for the three models while the left panel of Fig. 目 shows the angular power spectrum for the same models. The expression for $\Theta^{i s w}$ is the ISW contribution to the total anisotropies as defined in $\mathrm{Hu}$ and Sugiyama [55] eq.11. 

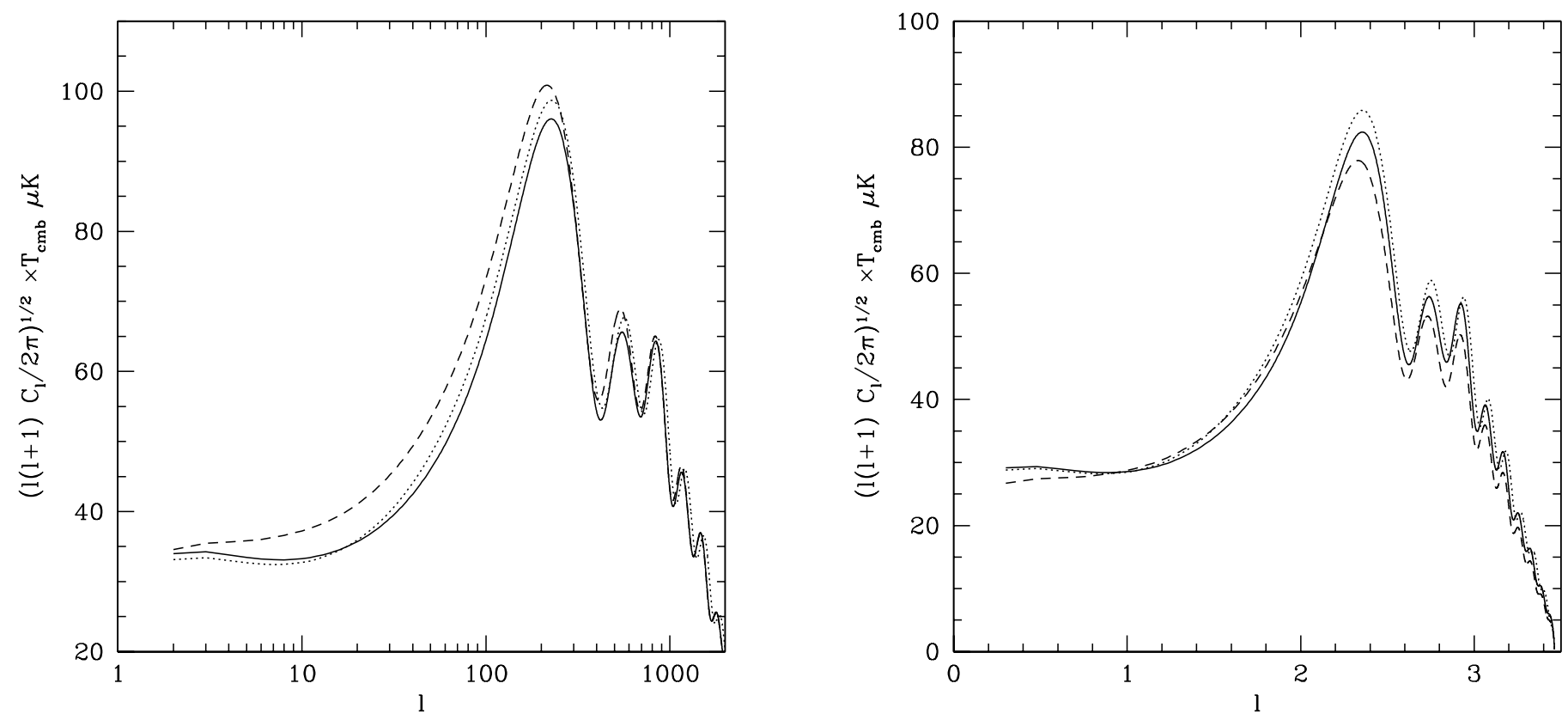

FIG. 7. The CMB anisotropies for the models of Fig. 2. Shown on the left are the anisotropies with the same initial power spectrum and on the right after COBE normalizing. Due to the strong ISW the anisotropies of the Brane model (dashed) are suppressed after normalization.

The ISW effect is very important for the Brane model (shown in all the relevant figures with a dashed curve). If what we saw today was just primary anisotropies then this model should have had the highest first peak of all three models. What we see however is exactly the opposite: it has the lowest peak. This is completely due to a very strong ISW which is shown on the right panel of Fig. 5. The ISW for the Brane model boosts power at large scales $(l<100)$ compared to the other models. Therefore it will have a direct effect on COBE normalization(or in fact any other kind of normalization which includes points at those scales)- the anisotropies have to be scaled down to fit COBE. What happens physically is that to get the observed power, one has to use a smaller amplitude for the initial power spectrum. This makes the the ratio of the first peak to the spectrum at COBE scales smaller in the case of the Brane model. Normalizing to COBE then suppresses the small scale anisotropies. So even though the model had the highest peak at the LSS, it now has the smallest.

The second effect of the very strong ISW on the Brane model is to shift the first acoustic peak to larger scales. This is though of lesser magnitude than the same effect caused by the smaller angular diameter distance.

Finally we also have gravitational lensing on the CMB photons. This is more significant on smaller scales. In the case of polarization the effect is smaller than $10 \%$ and for the temperature anisotropies even smaller [57]. The effect is a smearing of the peaks due to mixing of $l$-values without destroying the overall structure of the peaks. We calculate it using the linear evolution method of CMBFAST and assuming that quintessence will have the same effect on lensing as a pure cosmological constant. This is reasonable since like a cosmological constant, quintessence doesn't cluster and therefore its power spectrum does not contribute to lensing. The quintessence power is actually much smaller than the photon or neutrino power which themselves make a vary small contribution and are neglected.

\section{Position of the peaks}

The position of the peaks depends on two quantities, the sound horizon and the angular diameter distance to LSS. Both are affected by the presence of quintessence.

First lets consider the sound horizon. Even though the baryon density is kept the same, hence the speed of sound is the same in all three models, the time to the LSS is different due to the different expansion rate. Hence the sound horizon given by

$$
r_{s}=\int_{0}^{\tau_{*}} c_{s} d \tau
$$



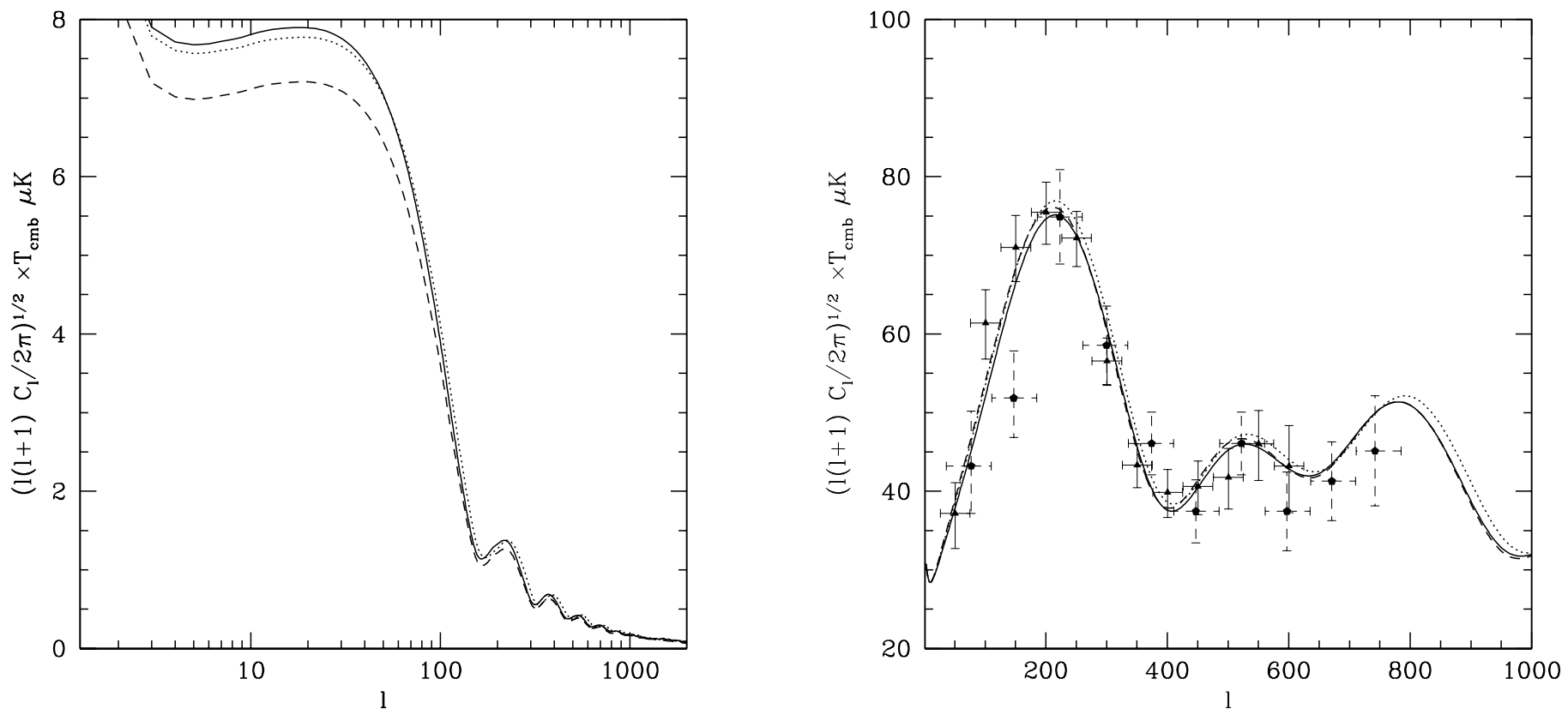

FIG. 8. LEFT: Tensor $C_{l}$ s for cosmological constant and the two quintessence models. All models have $h=0.65, \Omega_{b}=0.053$ $\Omega_{c}=0.247, \Omega_{\phi}=0.7, n_{s}=0.98, n_{t}=-0.02$ and ratio of tensor to scalar quadrupole of 0.1. Shown is a $\Lambda$ CDM(solid), AS (dotted) with $\lambda=5, B=54.4057, A=.01$ and Brane (dashed) model with $\lambda=5, B=56.10425, A=.01, C=1$ and $D=0.1$. RIGHT: The CMB anisotropies for the more realistic models of Fig. 4(Right panel). The effects discussed in the text are still visible but not as strong. Shown are the BOOMERanG98(solid) [58] and MAXIMA(dashed) [59] data.

is $\operatorname{different}\left(\tau_{*}\right.$ is the time at last scattering). More specifically it is inversely proportional to $H$ which means that quintessence has a smaller sound horizon. This shifts the anisotropies at LSS(in $k$-space) to smaller scales. Greater $H$ also makes the time at LSS $\tau_{*}$ smaller.

Next we have the angular diameter distance to LSS which is smaller in the case of quintessence. For the AS model it is not much smaller than a $\Lambda$ model since the universe starts accelerating at around the same time in both models. In the case of the Brane model however that universe is younger and therefore the angular diameter distance to LSS, smaller. In fact the more quintessence we have during the history of the universe, the younger it is since the universe starts accelerating later. If we have enough quintessence it doesn't accelerate at all. This appears to be counterintuitive. Remember however that in order to have more quintessence around one has to make the potential shallower. If it is shallow enough the field just rolls through.

From the analysis of 55] the difference in $l$ between peaks is given by

$$
\delta l=\pi \frac{\tau_{0}-\tau_{*}}{r_{s}}
$$

where $\tau_{0}$ is the time today. For the models of the right panel of Fig. 7 we get 292, 297 and 293 which is in very good agreement with the actual numerical result. The AS model has roughly the same angular diameter distance as the $\Lambda \mathrm{CDM}$ model but a smaller sound horizon. This makes the peak separation larger. The Brane model has the smallest sound horizon of all, but it also has the smallest angular diameter distance to the LSS since the universe is younger. The two roughly cancel each other and give roughly the same peak separation as in a $\Lambda$ CDM model.

Then we have the position of the first acoustic peak. The AS model has a first peak roughly at the same scale as the $\Lambda$ model. The Brane model however has the first peak shifted to larger scales. This is a combination of two effects. On is the much smaller angular diameter distance and the other is the very strong ISW.

\section{Tensor modes}

As mentioned previously, the scalar field has no shear and therefore the only effect it has on the tensor modes is through the background evolution. The tensor anisotropies are shown in Fig, 8 . 

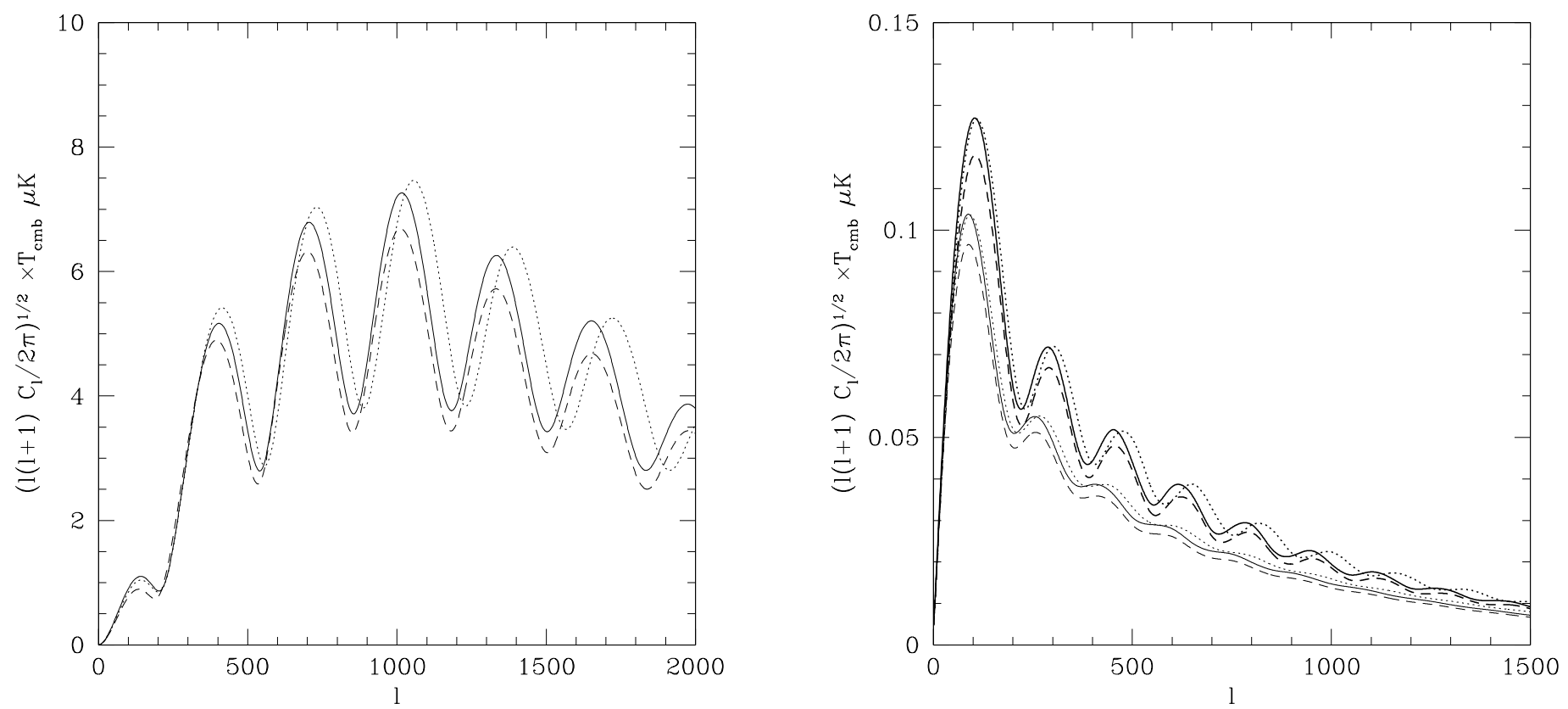

FIG. 9. LEFT: E-type polarization for the same models as Fig. 2. The strong ISW in the case of the Brane model suppresses indirectly the polarization spectrum.

RIGHT: Tensor E-type(heavy curves) and B-type(light curves) polarization for the models same models as Fig.8(left panel). Since there is no ISW the difference in the AS and $\Lambda$ models is just in the frequency of oscillation. For the Brane model we do have however the indirect effect of a strong ISW through normalization of the scalar $C_{l}$ s amplitude

The amplitude of the tensor modes is in general much less than the scalar amplitude and the only important contribution comes at large scales. Even though they don't affect the temperature anisotropy much they are still important as they create B-type polarization which might be observed.

The main effect is the strong ISW of the Brane model which suppresses the anisotropies at very large scales through the mechanism discussed in the earlier subsection. In the case of the AS model, the ISW is not as strong, so the model still retains anisotropies of roughly the same amplitude as a $\Lambda$ model. Finally we have the shifting of the oscillation pattern due to the different sound horizon and angular diameter distance just like the scalar case.

\section{E. Polarization}

Polarization depends only on the physics at the LSS, unlike the temperature anisotropy which also depends on the evolution since the LSS. It is therefore a direct probe of the physics at the LSS. For a review of polarization see [60]

E-type polarization for scalar modes is shown in Fig. 9. The first feature we observe is a difference in the position of the peaks. This is due to the same effects as in the scalar $C_{l}$ case, i.e. the sound horizon and angular diameter distance.

The heights of the peaks are affected by the driving effect on the local quadrupole at LSS. Therefore in the left panel of Fig. 9 we see again as expected that the AS model has the largest amplitude followed by the $\Lambda$ model. The Brane model should have had the largest amplitude but we have again the indirect effect of the ISW on the normalization which suppresses the whole thing. This happens again in this case because we normalized to COBE- the mechanism of suppression being the same as the one described in the scalar $C_{l}$ case.

B-type polarization is shown on the right of Fig.9. To get a sizable B-type polarization we used slightly different cosmological parameters(shown in the caption) from the ones used so far, basically changing the spectral indices and having a non-zero tensor to scalar quadrupole amplitude. The difference in the AS and $\Lambda$ models is just in the frequency of oscillation. For the Brane model we do have however the indirect effect of a strong ISW through normalization of the temperature anisotropies amplitude(same thing as in the E-polarization). We should note however that lensing can also produce B-type polarization but we don't discuss it here since in the case of these models quintessence can be treated like a cosmological constant as far as lensing is concerned.

Finally we have the polarization-temperature cross correlation shown in Fig.10. The importance of the cross 

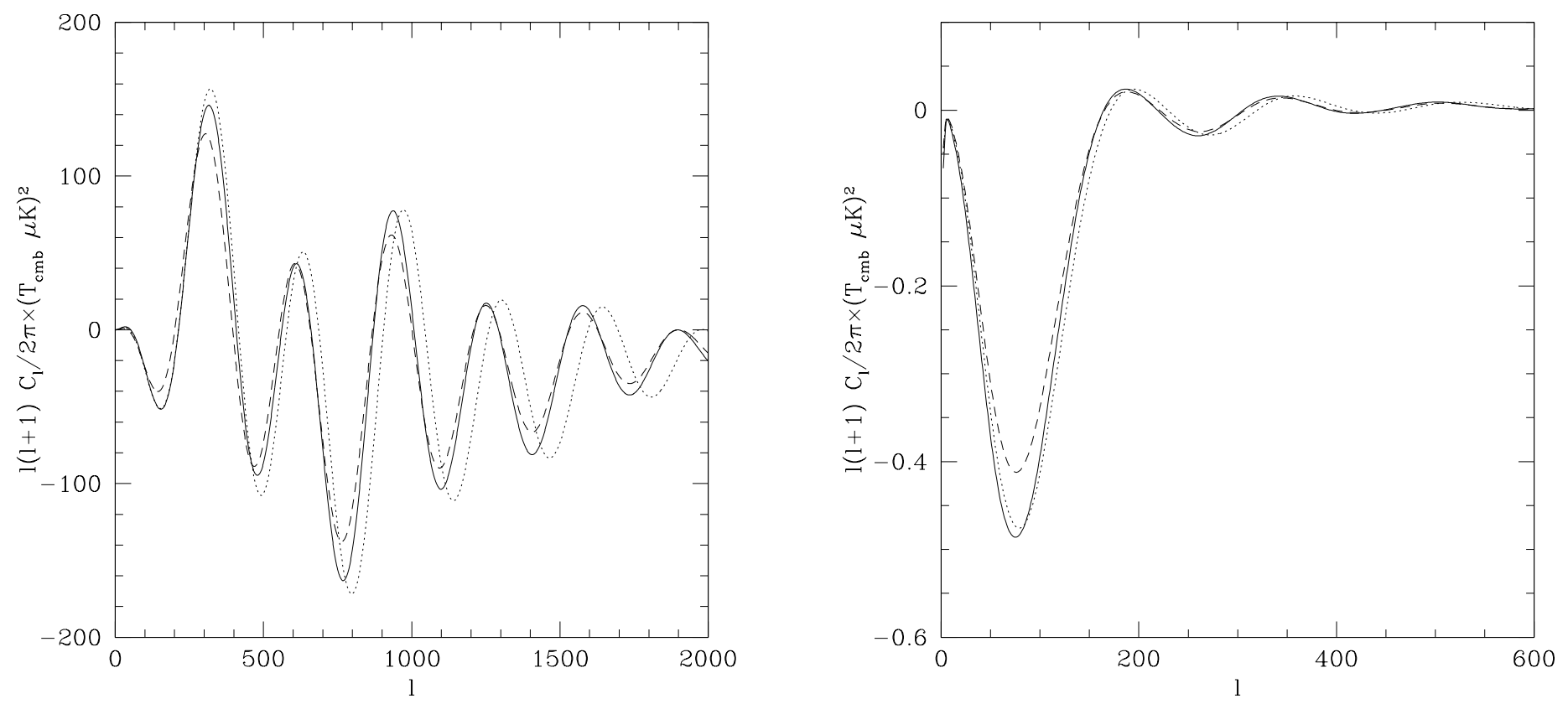

FIG. 10. Cross correlation of temperature and polarization. On the left we have scalar cross correlation for the same models as Fig. 1. On the right we have tensor cross correlation for the same models as Fig. 8 In both cases we see a strong suppression of the correlation amplitude for the Brane model due to the ISW effect.

correlation has been discussed in [61] for scalar modes and [62 for tensor modes. Again we see the different oscillation frequencies due to the different sound horizons and angular diameter distances just like the temperature anisotropies case, for both the scalar and tensor modes. In the case of the Brane model the amplitude of the correlation is smaller simply because in this model the temperature anisotropies (after normalization) are smaller compare to the other models. Have we kept the same initial power spectrum for all models the Brane model would have had the highest correlation. In the scalar case, on scales between $l>200$ and $l<1000$ we have the driving effect again giving more correlation to the quintessence models. Moreover for $l<600$ we also have the ISW boosting the correlation for quintessence. At very small scales the correlation amplitude tends to be the same in all models.

\section{THE MAGNITUDE-REDSHIFT RELATION}

Before concluding we comment briefly on the magnitude-redshift relation for the brane model. The magnituderedshift relation $m(z)$ for a standard candle of fixed fiducial absolute magnitude is an essential tool for pinning down the nature of the cosmic acceleration. Different quintessence models will produce different functions $m(z)$ which can be discriminated using data from, for example, type Ia supernovae (see for example [63]). When considering possible future probes of $m(z)$ it is essential to subject the space of possible models to the constraint that the models give a realistic account of cosmic structure. In particular, one can expect the structure-suppressing nature of quintessence to result in substantial constraints on the significance of quintessence at $z$ greater than around unity since structure needs to have a chance to form [64. It is therefor intriguing that that the Brane model (which gives a good fit to cosmic structure data) has some pretty interesting features in $m(z)$.

Figure 11 shows $\Delta m(z)$ for the Brane model (identical to the model shown in figure 1 ), a model for which the dark energy has an equation of state $p=w \rho$ with constant $\mathrm{w}=-0.9559$, and simulated binned data representing 1900 supernova events as might be provided by the proposes SNAP satellite [66]. The $m(z)$ curve for a pure cosmological constant model (which was used to produce the simulated data) is subtracted from $m(z)$ for the other models to get $\Delta m(z)$. Except for the choice of theoretical models shown, Fig. 11 is identical to Fig. 2 in [63], where details of its construction can be found.

The $\Delta m(z)$ curves for the two dark energy models are essentially identical up to $z \approx 1.3$ at which point they diverge. As discussed in [63, 65] there is no question that a SNAP-type data set will have a high impact on our ability to discriminate among models of the dark energy. Our work on the Brane model illustrates how even with a high quality SNAP-class data set in hand, there could be exciting opportunities to further discriminate among realistic 
models if a standard candle could be found that is effective out to higher $z$.

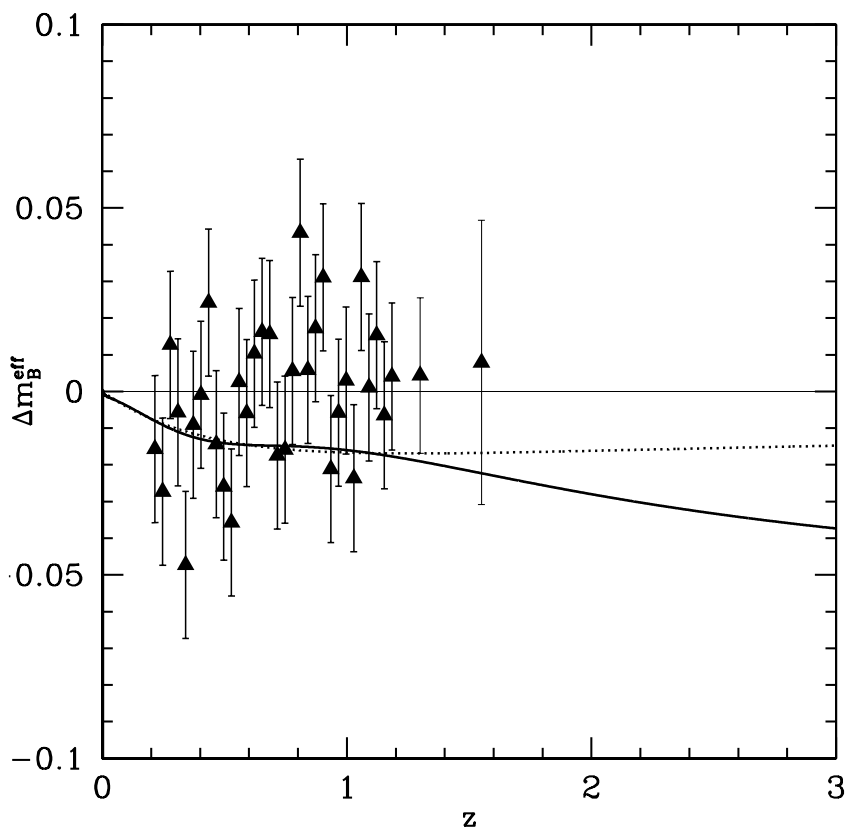

FIG. 11. $\Delta m(z)$ for the Brane model (heavy curve) and a $w=$ const. $=-0.9559$ model (dashed curve), along with simulated SNAP data. The curves are essentially identical up to $z \approx 1.3$ at which point they diverge. If a standard candle could be found that is effective at high redshifts it could play a significant role in discriminating among these types of models (We thank J. Weller for producing this figure).

\section{CONCLUSIONS}

We have presented a detailed investigation of a class of quintessence models motivated by our earlier work [19]. These models employ a particular mechanism (the exponential potential with a prefactor) which allows realistic models to be produced with all potential parameters $O(1)$ in Planck units. The potentials have a reasonable chance of developing strong theoretical foundations in brane theory or other theories with extra dimensions. The work presented here has taught us that these models also have an interesting and potentially observable impact on the formation of cosmic structure. The same mechanism that makes these models attractive from the point of view of fundamental physics causes the quintessence to play a much more significant role throughout the history of the Universe. This feature leads to interesting effects on the microwave background anisotropies, the matter power spectrum and the magnitude redshift relation that result in potentially observable differences from the predictions of other dark energy models. We have extensively examined the physical causes and the nature of these effects.

To illustrate the possibilities, Fig. 12 shows the full CMB Temperature anisotropy power spectrum for the Brane (right) and AS (left) models, containing all the effects discussed in this paper. Each plot also shows the $\Lambda$ model which best mimics the corresponding quintessence model. The lower panel of each plot shows that despite the closeness of the two curves, they are potentially differentiable by the MAP data (and of course we will eventually have much more than just the MAP data set).

One issue which still needs to be addressed is the degree to which the signals we have discovered can realistically be differentiated from all possible mimicking behavior due to the dependence of the predictions on a range of cosmological parameters (the mimicking $\Lambda$ models in Fig. 12 were produced "by hand", by making a thorough but not completely exhaustive exploration of all possible parameters). This paper lays the groundwork for such a project. The full impact of our results will not be know until this this issue is addressed in a more systematic way.

Still, it is quite interesting that our quintessence models give predictions that fit all existing constraints, and which leave a potentially unique set of signals that could be observed with future experiments. 

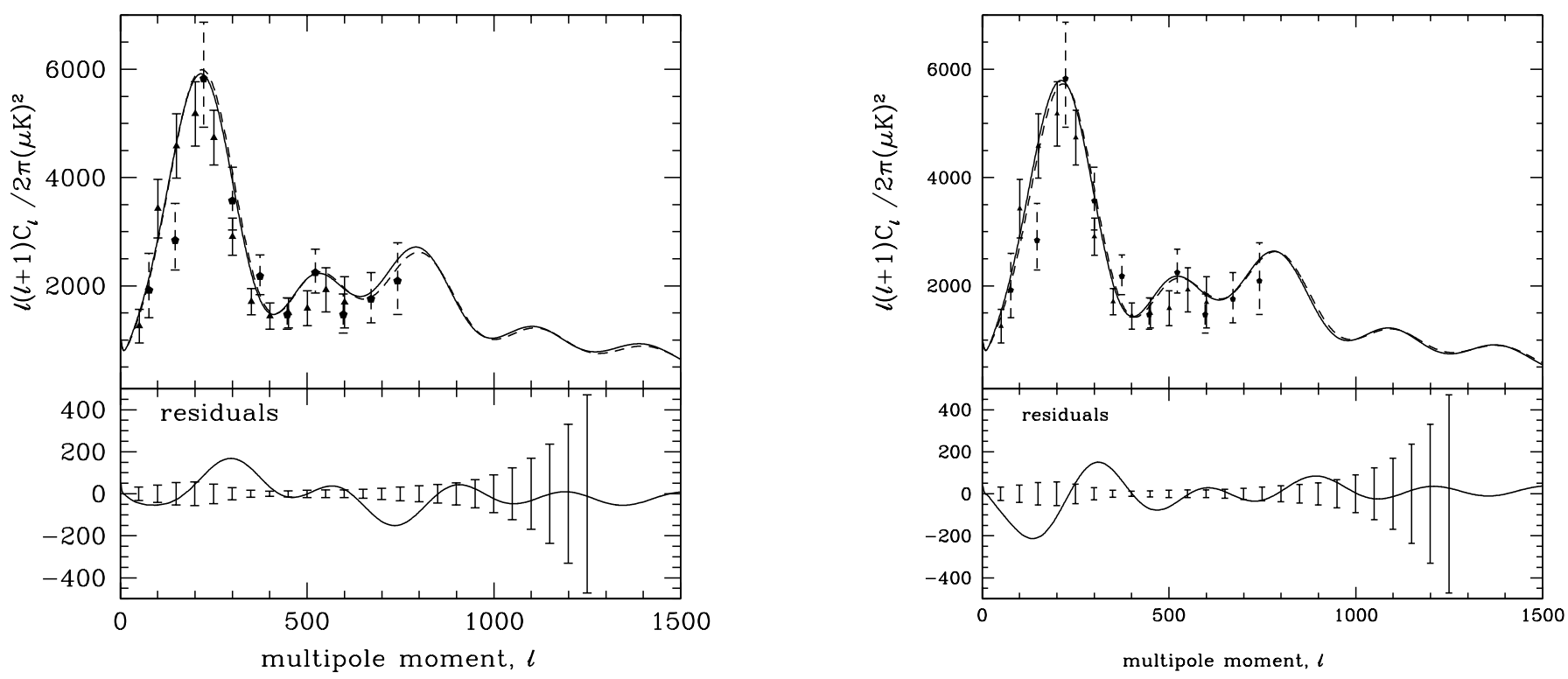

FIG. 12. A possible future comparison of the AS and Brane models with a $\Lambda$ model using MAP data. On the left we have the AS model of the right of Fig 4 compared with a $\Lambda$ model with $h=0.75, \Omega_{\Lambda}=0.7005, \Omega_{b}=0.053, \Omega_{c}=0.2465$ and $\tau=0.1$. On the right we have the Brane model of the right of Fig. 4 compared with a $\Lambda$ model with $h=0.75, \Omega_{\Lambda}=0.66327, \Omega_{b}=0.053$, $\Omega_{c}=0.28373$ and $\tau_{\mathrm{opt}}=0.1$. The two $\Lambda$ models were chosen to match the first peak and at the same time the rest of the curve as close as possible. On the bottom of each figure we show the residuals of the two corresponding models with MAP error-bars (We thank L.Knox for providing the MAP error-bars).

\section{ACKNOWLEDGMENTS:}

We thank A. Lewin, B. Gold, M. Turner, Y.-S. Song, L.Wang and J. Weller for helpful conversations. A.A. thanks the Aspen Center for Physics and C.S. thanks International Center for Theoretical Physics where some of this work was completed. This work was supported by DOE grant DE-FG03-91ER40674 and U.C. Davis. The CMB and matter power spectra used in this work were generated by a modified version of CMBFAST [67.

[1] N. Bahcall, J. Ostriker, S. Perlmutter and P. Steinhardt, Science 284, 1481, (1999).

[2] Jaffe et al. astro-ph/0007333.

[3] T. Banks, M. Berkooz, G. Moore, S. H. Shenker and P. J. Steinhardt, Phys. Rev. D 523548 (1995).

[4] J. Frieman, C. Hill, A. Stebbins and I. Waga Phys. Rev. Lett. 752077 (1995).

[5] K. Coble, S. Dodelson and J. Frieman, Phys. Rev. D 551851 (1997).

[6] R. Caldwell and P. Steinhardt, Phys. Rev. D 576057 (1998).

[7] L. Wang and P. Steinhardt, Astrophys. J. 508483 (1998).

[8] R. Caldwell, R. Dave, P. Steinhardt Phys. Rev. Lett. bf 801582 (1998).

[9] G Huey, L. Wang, R. Dave, R. Caldwell and P. Steinhardt, Phys. Rev. D 59 (1999) 063005.

[10] P. Steinhardt, L. Wang and I. Zlatev, Phys. Rev. D 59123504 (1999).

[11] I. Zlatev, L. Wang, and P. Steinhardt, Phys. Rev. Lett. 82896 (1999).

[12] C.-P. Ma, R. Caldwell, P. Bode, and L. Wang, Astrophys. J. Lett. 521 L1-L4 (1999).

[13] L. Wang, R. Caldwell, J. Ostriker and P. Steinhardt, astro-ph/9901388.

[14] T. Chiba, Phys.Rev. D 60 (1999) 083508.

[15] V. Sahni and A. Starobinsky astro-ph/9904398 to appear in: International Journal of Modern Physics D.

[16] A. Masiero, M. Pietroni, F. Rosati hep-ph/9905346.

[17] F. Perrotta, C. Baccigalupi and S. Matarrese astro-ph/9906066. 
[18] I. Zlatev and P. Steinhardt, Phys.Lett. B459 (1999) 570-574

[19] A. Albrecht and C.Skordis, Phys.Rev.Lett 84, 2076, (2000).

[20] T. Matos, L. A. Urena-Lopez, Class.Quant.Grav. 17 (2000) L75-L81

[21] L. A. Urena-Lopez, T. Matos, astro-ph/0003364

[22] E.J. Copeland, N.J. Nunes, F. Rosati, hep-ph/0005222

[23] T. Matos, L. A. Urena-Lopez, astro-ph/0006024

[24] C. Armendariz-Picon,V. Mukhanov and P.J. Steinhardt, astro-ph/0006373

[25] K. Choi Phys.Rev. D62 (2000) 043509

[26] R. Battye, M. Bucher, D. Spergel, astro-ph/9908047; M. Bucher, D. Spergel ,Phys. Rev. D 60 (1999) 043505.

[27] P. Viana, A. Liddle, Phys. Rev. D 57 (1998) 674-684.

[28] P. Binetruy Phys. Rev. Lett.D60 (1999) 063502;

[29] P. Brax and J. Martin, Phys.Rev.D61 (2000) 103502

[30] P. Brax, J. Martin, A. Riazuelo, astro-ph/0005428

[31] S. Dodelson, M. Kaplinghat, E. Stewart astro-ph/0002360

[32] S. Perlmutter, M. Turner and M. White Phys.Rev.Lett. 83 (1999) 670-673

[33] J.J. Halliwell, Phys. Lett. B185 341 (1987).

[34] J. Barrow, Phys. Lett. B187 12 (1987).

[35] C. Wetterich, Astron. and Astrophy. 301321 (1995).

[36] B. Ratra and P. Peebles, Phys. Rev. D 373406 (1988).

[37] E. Copeland, A. Liddle and D. Wands, Ann. N. Y. Acad. Sci. 688647 (1993).

[38] T. Barreiro, B. de Carlos, E.J. Copeland , Phys.Rev. D 58 (1998) 083513; E. Copeland, A. Liddle and D. Wands, Phys. Rev. D 57 (1998) 4686-4690.

[39] P. Ferreira, M. Joyce Phys. Rev. D 58023503 (1998).

[40] N. Arkani-Hamed et al. astro-ph/0005111

[41] R. Bean and J. Magueijo astro-ph/0007199

[42] A. Hebecker and C. Wetterich hep-ph/0003287

[43] D. Huterer and M. S. Turner Phys. Rev. D 60, 081301 (1999)

[44] J. Barrow, R. Bean, J. Magueijo, astro-ph/0004321

[45] J. Weller, astro-ph/0004096

[46] G.Dvali and H.Tye, Phys.Lett. B450 (1999) 72-82

[47] e.g. K. Olive, G. Steigman and T. Walker, astro-ph/9905320.

[48] J.M. Bardeen, Phys. Rev. D 22, 1882 (1980)

[49] H. Kodama and M. Sasaki, Prog. Theo. Phys. Suppl. 781 (1984)

[50] V.F. Mukhanov, H.A. Feldman and R.H. Brandenberger, Phys. Rep. 215 203-333 (1992)

[51] C.P. Ma and E. Bertschinger, Astrophys. J. 455 7-25 (1995)

[52] A. J. S. Hamilton and M. Tegmark, astro-ph/0008392

[53] A. J. S. Hamilton, M. Tegmark and N. Padmanabhan, MNRAS 317, L23-L27 (1 Sep 2000)

[54] W.Hu and N.Sugiyama, Astrophys.J. 471 542-570 (1996)

[55] W.Hu and N.Sugiyama, Astrophys.J. 444489 (1995)

[56] W.Hu and N. Sugiyama, Phys. Rev. D 50627 (1994)

[57] M. Zaldarriaga, U. Seljak Phys.Rev.D 58023003 (1998)

[58] A.E. Lange et al, astro-ph/0005004

[59] A. Balbi et al, astro-ph/0005124 submitted to ApJ.

[60] W.Hu and M.White, New Astron. 2323 (1997)

[61] D. Coulson, R. G. Crittenden, N.G. Turok, astro-ph/9406046

[62] R.G. Crittenden, D. Coulson, N.G. Turok, Phys.Rev. D 52 5402-5406 (1995)

[63] J. Weller and A. Albrecht astro-ph/0008314, submitted to PRL

[64] This point

been emphasized by M. Turner in his talk at the PASCOS99 meeting (http://pc90.ucdavis.edu/pascos99.html and in discussions during the Aspen Winter conference "Way beyond the standard model" Jan-Feb 2000.

[65] J. Weller and A. Albrecht in preparation.

[66] snap.lbl.gov

[67] U. Seljak and M. Zaldarriaga, Astrophys. J. 469437 (1996). 